March 1, 1997

Brussels

\section{Meeting of the Belgian Royal Society for Dermatology and Venereology}

\author{
Editor: Prof. G.E. Piérard
}

\section{Contents}

Shielded Corneosurfametry and

Corneoxenometry: Novel

Bioassays for the Assessment of Skin Barrier Products

V. Goffin, C. Piérard-Franchimont,

G.E. Piérard

The in vitro and in vivo

Production of a 31.5-kD

Keratinolytic Subtilase from

Microsporum canis and the

Clinical Status in Naturally Infected Cats

B.R. Mignon, A.F. Nikkels,

G.E. Piérard B.J. Losson.

Low-Productive Alpha-Herpesviridae Infection in Chronic Lichenoid Dermatoses

A.F. Nikkels, C. Sadzot-Delvaux,

B. Rentier, C. Piérard-Franchimont,

G.E. Piérard.

Role of Viral Agents in the

Pathogenesis of

Kaposi's Sarcoma

Th. Simonart, J.C. Noel,

J.P. Van Vooren, D. Parent 447
Breast and Lung Cancers in Two Cyclosporin-A-Treated Psoriatic Women

P. Paquet, G.E. Piérard 450

Acne neonatorum and Familial Hyperandrogenism

C. Bekaert, M. Song, A. Delvigne .... . 453

Granulomatous Vulvitis,

Granulomatous Cheilitis:

A Single Diagnosis?

S. Thiriar, E. Deroux, N. Dourov,

L. Evrard, M.O. Peny, P. Simon,

D. Parent.

Tuberculous Inguinal and

Crural Lymph Nodes

H. Binet, J.P. Van Vooren, J.P. Thys,

M. Heenen, D. Parent 459

Toxic Epidermal Necrolysis

Associated with Treatment for

Preterm Labor

N. Claessens, L. Delbeke, J. Lambert,

L. Matthieu, C. Lafaire,

E. Van Marck
The Schöpf-Schulz-Passarge Syndrome

P. Verplancke, L. Driessen,

P. Wynants, J.M. Naeyaert . .

Hypomelanosis of Ito

I. Haenen, L. Vanquickenborne,

S. Kerre, H. Degreef .

Contact Allergy to Resorcinol

Monobenzoate

K. Ongenae, L. Matthieu,

L. Constandt, E. Van Hecke ...... . . . 470

Ketoconazole Shampoo:

Effect of Long-Term Use in

Androgenic Alopecia

C. Piérard-Franchimont, P. De Doncker,

G. Cauwenbergh, G.E. Piérard. 